\title{
Characterization of Poly $(\beta$-L-malic acid) Produced by Aureobasidium sp. A-91
}

\author{
Naoki NagATa, ${ }^{\dagger}$ Tadaatsu Nakahara, Takeshi TABUChI, ${ }^{\dagger \dagger}$ Reiko Morita, ${ }^{*}$ \\ Jason R. BREWER, ${ }^{*}$ and Shouei FuJISHIGE* \\ Institute of Applied Biochemistry, University of Tsukuba, Tennoudai 1-1, Tsukuba 305, Japan \\ ${ }^{*}$ Research Institute for Polymers \& Textiles, Higashi 1-1, Tsukuba 305, Japan
}

(Received October 9, 1992)

\begin{abstract}
In a previous paper, black yeast was found to produce poly(L-malic acid) in large yield by fermentation with glucose as the carbon source. Based upon characteristic NMR spectra, the primary structure of this polymer was elucidated as poly $(\beta$-L-malic acid $)$ having branched chains. Thermal analysis by DSC shows neither $T_{\mathrm{g}}$ nor $T_{\mathrm{m}}$ below its decomposition temperature $T_{\mathrm{d}}$ on the solid state polymer.

KEY WORDS NMR / DSC / Auto-Catalytic Cleavage / Enzymatic Assay /

Oligomer /
\end{abstract}

Poly(malic acid) is well known as a product by Penicillium cyclopium, Physarum polycephalum, and Aureobasidium sp. ${ }^{1}$ In the case of poly(malic acid), there are three different possible isomers:

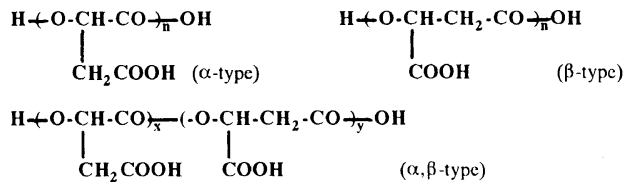

Poly(malic acid)s of a different structure have been synthesized in vitro via direct condensation reaction of malic acid, or by the condensation reaction of $\alpha$ - or $\beta$-malic acid benzyl ester to give a specified isomeric polymer followed by debenzylation, ${ }^{2,4}$ but the molecular weight of the polymers thus obtained were limited (less than 4000).

As reported in our previous paper, ${ }^{1}$ Aureobasidium sp. strain A-91 isolated as black yeast gave rather high molecular weight poly-
(L-malic acid) extracellularly in the course of fermentation with glucose as the carbon source. In the culture broth, pullulan coexisted with this polymer but the poly(malic acid) was easily recovered as its $\mathrm{Ca}$-salt by precipitation with methyl alcohol.

In this paper, we report the elucidation of the primary structure of poly(L-malic acid), based on both the ${ }^{1} \mathrm{H}$ and ${ }^{13} \mathrm{C}$ NMR spectra which were recorded in aqueous solution. NMR spectroscopy was also used to study the auto-catalytic cleavage behavior of the polymer in aqueous solution. Thermal properties of the solid state poly(L-malic acid) were also examined by DSC.

\section{EXPERIMENTAL}

\section{Source of Polymalate}

In the presence of Aureobasidium sp. A-91 isolated in the form of black yeast (the best producer of poly(malic acid)) a culture broth

$\dagger$ To whom all correspondence should be sent.

${ }^{\dagger \dagger}$ Present address: Department of Bioscience and Biotechnology, Aomori Univ., Kobata 030, Japan. 
was prepared by using a basal medium containing $12 \%$ of glucose as the carbon source. ${ }^{1}$ During the course of fermentation (one week at $25^{\circ} \mathrm{C}$ ), the $\mathrm{pH}$ of the medium was maintained at $\mathrm{pH} 6.8$ by adding $\mathrm{CaCO}_{3}$.

\section{Isolation and Purification of Poly(malic acid)}

The culture broth thus obtained was filtrated through a layer of kieselguhr. After warming up the filtrate $(2.31, \mathrm{pH} 6.4)$ to $50^{\circ} \mathrm{C}$, methyl alcohol $(0.81)$ was added dropwise with stirring to precipitate pullulan ( $c a$. $2 \mathrm{~g}$ ) which was removed as a stringy solid by filtration. Methyl alcohol (2.71) was further added dropwise and the mixture was left overnight with mild stirring at room temperature. The resulting precipitate $(c a .140 \mathrm{~g})$ of Ca-salt of poly(L-malic acid) was collected by decantation.

For the molecular weight fractionation, the Ca-salt of poly(L-malic acid) $(80 \mathrm{~g})$ was dissolved into deionized water (1 1$)$ and methyl alcohol $(0.5 \mathrm{l})$ was added dropwise at room temperature to give the highest molecular weight fraction (Fraction 1) (ca. $48 \mathrm{~g}$ ). Further amount of methyl alcohol (0.31 and 0.7 l) were consecutively added to give Fractions 2 and 3 (25 g and ca. $4 \mathrm{~g}$, respectively).

Of these fractions, Fraction 1 had the highest molecular weight (10000) and was used in subsequent experiments for characterization.

To obtain Ca-ion freed poly(L-malic acid), Faction 1 was dissolved into deionized water and treated with Amberlite IR-120B $\left(\mathrm{H}^{+}\right)$. The aqueous solution thus treated was then lyophilized and dried over $\mathrm{P}_{2} \mathrm{O}_{5}$ to a constant weight.

\section{Analytical Methods}

HPLC was carried out as previously described. ${ }^{1}{ }^{1} \mathrm{H}$ and ${ }^{13} \mathrm{C}$ NMR spectra were recorded on $\mathrm{D}_{2} \mathrm{O}$ solutions using JEOL-FX 100 and JEOL-GSX 270 spectrometers with DSS or TMS as internal standard references. Differential scanning calorimetry (=DSC) to observe the thermal properties of the solid state polymer was performed using a SEIKO DSC $220 \mathrm{C}+$ SSC 5200 instrument. All DSC samples were carefully sealed in aluminium pans to prevent water absorption. The temperature range scanned was from $25^{\circ} \mathrm{C}$ to $250^{\circ} \mathrm{C}$ (with a $10^{\circ} \mathrm{C} \mathrm{min}^{-1}$ heating rate). The optical rotation of cleavage products were assigned by enzymatic assay using the commercial kits of Boehringer Mannheim GMBH Biochemica, detecting specific activities of L-malate dehydrogenase or D-malate dehydrogenase, respectively.

\section{RESULTS AND DISCUSSION}

\section{Elucidation of the Primary Structure of Poly- (L-malic acid)}

In the case of poly(L-malic acid), there are three possible isomers, $\alpha$-, $\beta$-, and $\alpha, \beta$-type as described above. In Table I, previous assignments $^{2-4}$ of the NMR spectrum of poly(malic acid) in $\mathrm{D}_{2} \mathrm{O}$ are compared with that obtained in this report.

Discrepancies among the values of the chemical shifts from sample to sample might mainly be attributed to the differences in molecular weight and also to the low resolution of the spectra, because they could not detect any signals to be assigned to the terminal structures of the polymers.

It is clear from the literature values of chemical shifts presented in Table I that we are not able to assign each spectrum either to $\alpha$-type polymer or $\beta$-type polymer, consequently the assignment of primary structure was made indirectly. First of all, the NMR spectrum of the $\mathrm{D}_{2} \mathrm{O}$ solution of the Ca-salt of poly(L-malic acid) was compared with that of Ca-ion freed poly(L-malic acid) (Figure 1). We expected to observe a significant difference in the chemical shifts brought about by the ability of the Ca-ion freed polymer to behave as an expanded polyelectrolyte chain due to the intramolecular repulsive forces between the charged groups. In contrast, the Ca-salt of 
Table I. ${ }^{1} \mathrm{H}$ and ${ }^{13} \mathrm{C}$ NMR assignments of various poly(malic acid)s in $\mathrm{D}_{2} \mathrm{O}$

${ }^{1} \mathrm{H}$ NMR

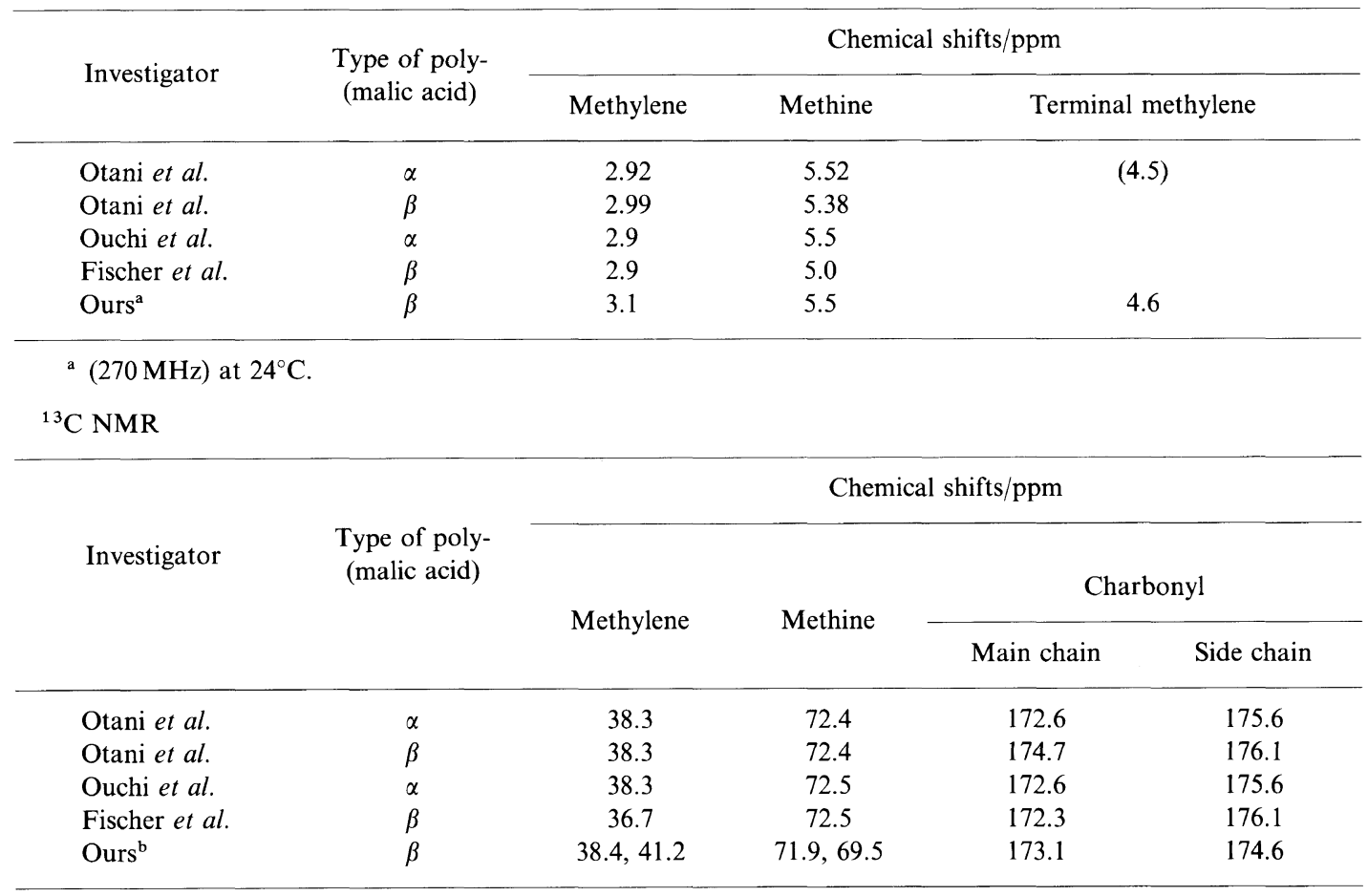

b $(67.94 \mathrm{MHz})$ at $24^{\circ} \mathrm{C}$.

the polymer behaves as a shrinked random coil in aqueous solution. It follows that the chemical shift of the methylene protons of $\alpha$-type polymer chain might be much affected than those of methylene protons of $\beta$-type polymer.

In Figures 1 and 2, it is seen that there is no significant difference in the values of the chemical shifts of the methylene unit in contrast to those of methine unit and of carbonyl unit. Therefore, the fact that a large induced change in chain conformation has little effect on the magnetic environment of the methylene protons, allows the assignment of a $\beta$-type structure to be made. Thus the primary structure of this poly(L-malic acid) was deduced as "poly $(\beta$-L-malic acid)."

The structure thus elucidated is supported by those data presented especially in Figures 4
(4) and 6 (3) which were assigned to those of monomeric L-malic acid and $\beta$-type conjugated L-malic acid in aqueous solutions.

In addition to the structural determination mentioned above, the fine structure of the individual signals of the methine- and methylene-protons were analyzed by NMR spectroscopy on aqueous solutions at different stages of auto-catalytic cleavage.

In Figure 3, comparison of individual signals appearing on the NMR spectra at different stages of cleavage enabled us to assign signals with fine structures to the terminal methine-, methylene-protons, to those of inner part of the polymer chain, and also to those of the structural unit next to or near to the terminal structure of the polymer chain. For example, using authentic sample (Spectrum (1)), methylene proton signals were observed at 3.0 and 

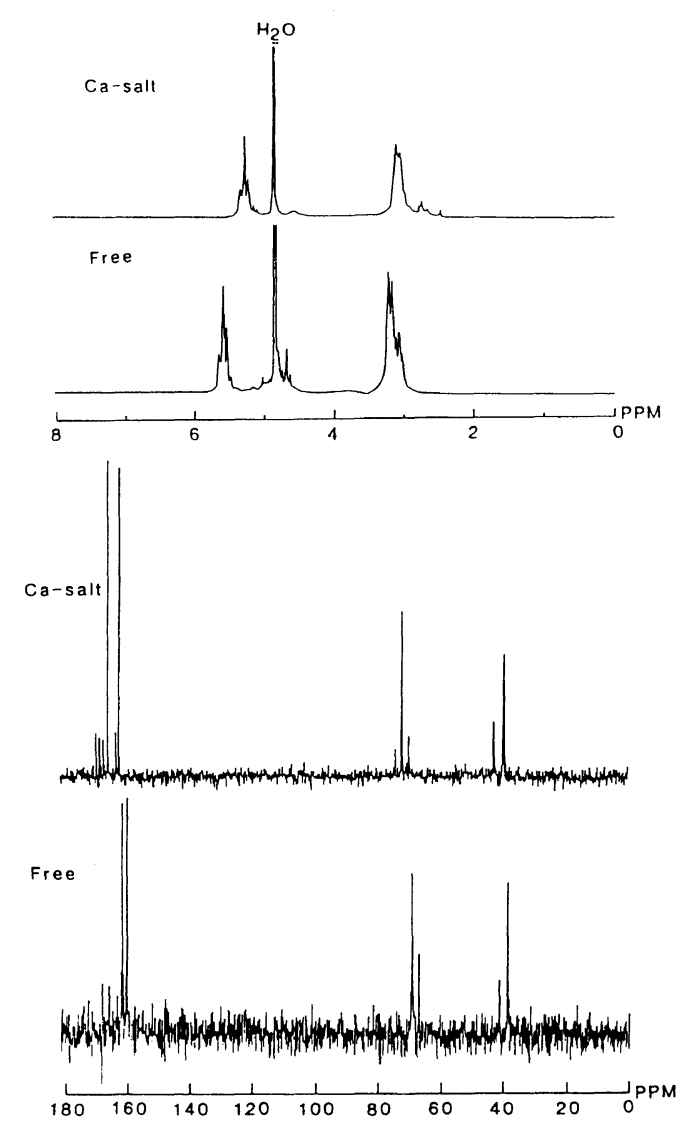

Figure 1. $270 \mathrm{MHz}{ }^{1} \mathrm{H}$ and $67.94 \mathrm{MHz}{ }^{13} \mathrm{C} \quad \mathrm{NMR}$ spectrum of the $\mathrm{D}_{2} \mathrm{O}$ solution of Ca-salt of poly(L-malic acid) at $24^{\circ} \mathrm{C}$ in comparison to that of $\mathrm{Ca}$-ion freed poly(L-malic acid).

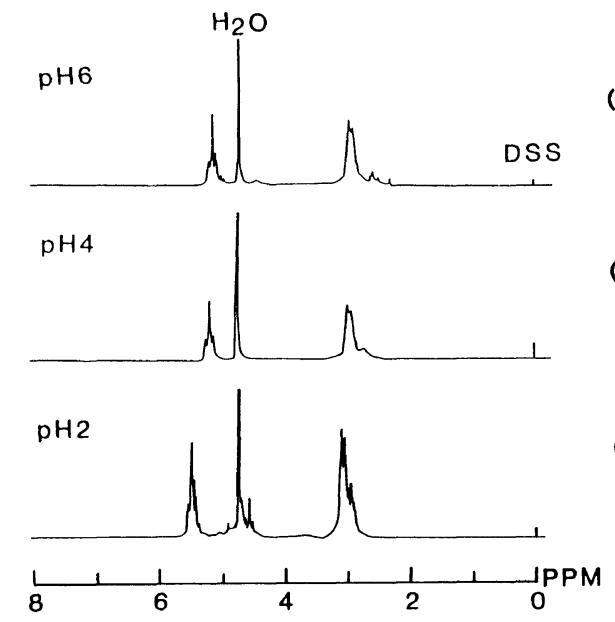

(3)

Figure 2. $270 \mathrm{MHz}{ }^{1} \mathrm{H}$ NMR spectra of the $\mathrm{D}_{2} \mathrm{O}$ solutions; effect of $\mathrm{pH}$ on aqueous solution of $\mathrm{Ca}$-salt of poly(L-malic acid) at $24^{\circ} \mathrm{C}$.

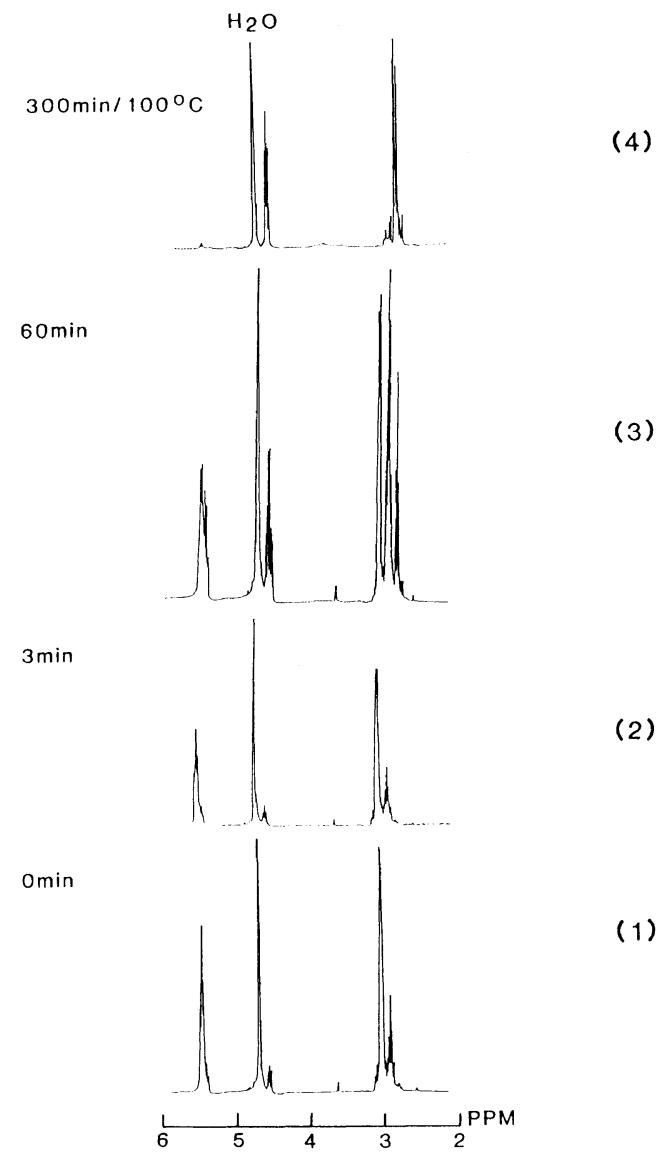

Figure 3. $270 \mathrm{MHz}{ }^{1} \mathrm{H}$ NMR spectra at different stage of auto-catalytic cleavage in $\mathrm{D}_{2} \mathrm{O}$ solution: $t=0 \mathrm{~min}$ compared with that of $t=3 \mathrm{~min}, t=60 \mathrm{~min}$, and $t=$ $300 \mathrm{~min}$.

$3.13 \mathrm{ppm}$ and the methine proton signal at $5.54 \mathrm{ppm}$. A methine signal at $4.7 \mathrm{ppm}$ was assigned as the terminal methine, while in Spectrum (4) $(t=300 \mathrm{~min})$, in addition to those from increasing amounts of resulting monomeric malic acid, signals assigned to be those of methine- and methylene-protons of the unit structure next to or near to the terminal structure as defined above.

In Spectrum (3) obtained after $60 \mathrm{~min}$ cleavage at $100^{\circ} \mathrm{C}$, the fine structure became more complex, but after comparing the spectrum with the others, assignment was made as follows: the signal intensity of both methine (5.5 ppm) and methylene (3.13 and $3.15 \mathrm{ppm})$ 
Characterization of Polymalate

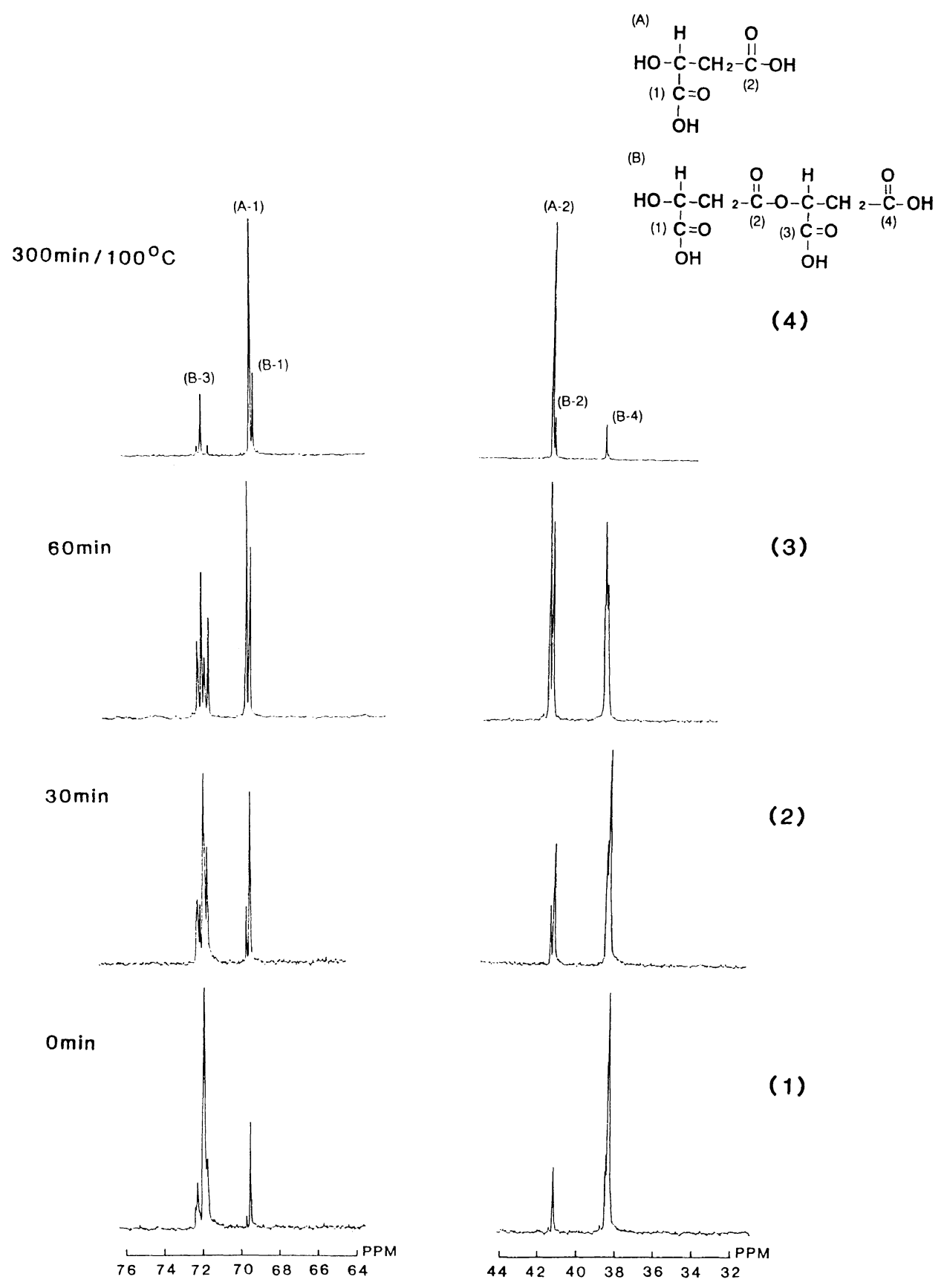

Figure 4. $67.94 \mathrm{MHz}{ }^{13} \mathrm{C}$ NMR spectra at higher magnetic field at different stages of auto-catalytic cleavage of poly(L-malic acid) in $\mathrm{D}_{2} \mathrm{O}$ solution: $t=0 \mathrm{~min}$ compared with that of $t=30 \mathrm{~min}, t=60 \mathrm{~min}$, and $t=300 \mathrm{~min}$. 
unit located at the inner part of the polymer chain decreased and the intensities of both methine $(4.6 \mathrm{ppm})$ and methylene $(2.8 \mathrm{ppm})$ protons of resulting monomeric malic acid increased. Remarkably, new signals at 3.0 and $4.7 \mathrm{ppm}$ were observed, and the signals were assigned to the oligomer of malic acid produced in the cleavage reaction.

In parallel to the $270 \mathrm{MHz}{ }^{1} \mathrm{H} \quad \mathrm{NMR}$ spectra, $67.92 \mathrm{MHz}{ }^{13} \mathrm{C} \mathrm{NMR}$ spectra were obtained on the same sample solution during the course of auto-catalytic cleavage as shown in Figure 4. The signals appearing at lower magnetic field (assigned to carbon atoms of $\mathrm{C}=\mathrm{O}$ units) were complicated at the first stage of the cleavage reaction as shown in Figure 6-(2). However, the signals became simple after $300 \mathrm{~min}$, giving two strong peaks due to monomeric malic acid and four peaks due to dimeric malic acid as shown in Figure 6-(3). On the basis of this assignment, the fine structure of the ${ }^{13} \mathrm{C}$ NMR spectrum at higher magnetic fields were assigned as shown in Figure 4-(4).

Table II compares the chemical shifts of these ${ }^{13} \mathrm{C}$ NMR spectra of monomeric- and dimeric-L-malic acid.

In Figures 4-(1) and -(2), the main peak at $38.40 \mathrm{ppm}$ is assigned to the carbon atom of the methylene unit located at the inner part of the polymer chain and the peak at $41.25 \mathrm{ppm}$ to the terminal methylene carbon. Similarly the main peak centered at $71.96 \mathrm{ppm}$ is assigned to those carbon of methine unit located at the inner part of the polymer chain and the peak at $69.57 \mathrm{ppm}$ is assigned to the terminal methine carbon. In contrast to those reported in previous papers, ${ }^{2-4}$ the signal assigned to the terminal structure of the authentic polymer was significant as compared to those of carbon atoms located at the inner part of the polymer chain. This means that the population of the terminal unit structure per polymer molecule is remarkably higher than those of precedents. Based on this fact, the primary structure of this $\operatorname{poly}(\beta$-L-malic acid) was
Table II. ${ }^{13} \mathrm{C}$ NMR assignments of monomericand dimeric-L-malic acid in $\mathrm{D}_{2} \mathrm{O}$

\begin{tabular}{lcc}
\hline & \multicolumn{2}{c}{ Chemical shifts/ppm } \\
\cline { 2 - 3 } & Methylene & Methine \\
\hline Monomer & 41.40 & 69.74 \\
Dimer & $38.59,41.25$ & $69.56,72.29$ \\
\hline
\end{tabular}

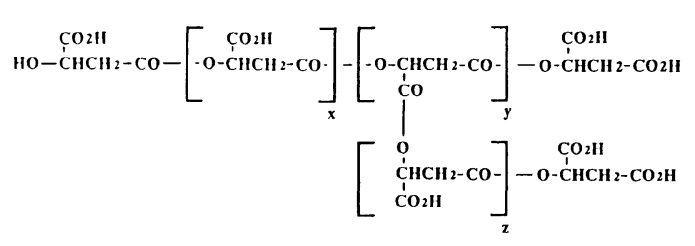

Figure 5. The primary structure of $\operatorname{poly}(\beta$-L-malic acid $)$ produced by Aureobasidium sp. A-91.

elucidated as not linear chain polymer but with branched chains as shown in Figure 5.

As it has already been shown in analysis of the fine structure of the ${ }^{1} \mathrm{H}$ NMR spectra, the main peaks observed at 173.0, 174.1, 174.8, and $179.0 \mathrm{ppm}$ of Spectrum (1) (Figure 6) are assigned to that of the carbonyl carbons while the two peaks at 177.4 and $179.4 \mathrm{ppm}$ of Spectrum (3) are assigned to those of resulting monomeric malic acid as indicated respectively. The fine structure in Spectrum (2) was also analyzed as indicated by comparing individual signals on each spectra. In other words, Spectrum (3) indicates that the major product after $300 \mathrm{~min}$ heating at $100^{\circ} \mathrm{C}$ is monomeric L-malic acid with a minor amount of oligomer (mainly dimer of $\beta$-L-malic acid).

\section{Thermal Properties of the Solid State Poly $(\beta-$ L-malic acid)}

Since this polymer is quite hygroscopic, all samples were carefully handled and sealed into aluminium pans under argon gas. It is seen in Figure 7 that neither $T_{\mathrm{g}}$ nor $T_{\mathrm{m}}$ of the solid state polymer were detected below the decomposition temperature starting at about $185^{\circ} \mathrm{C}$ as a distinct endothermic reaction. 


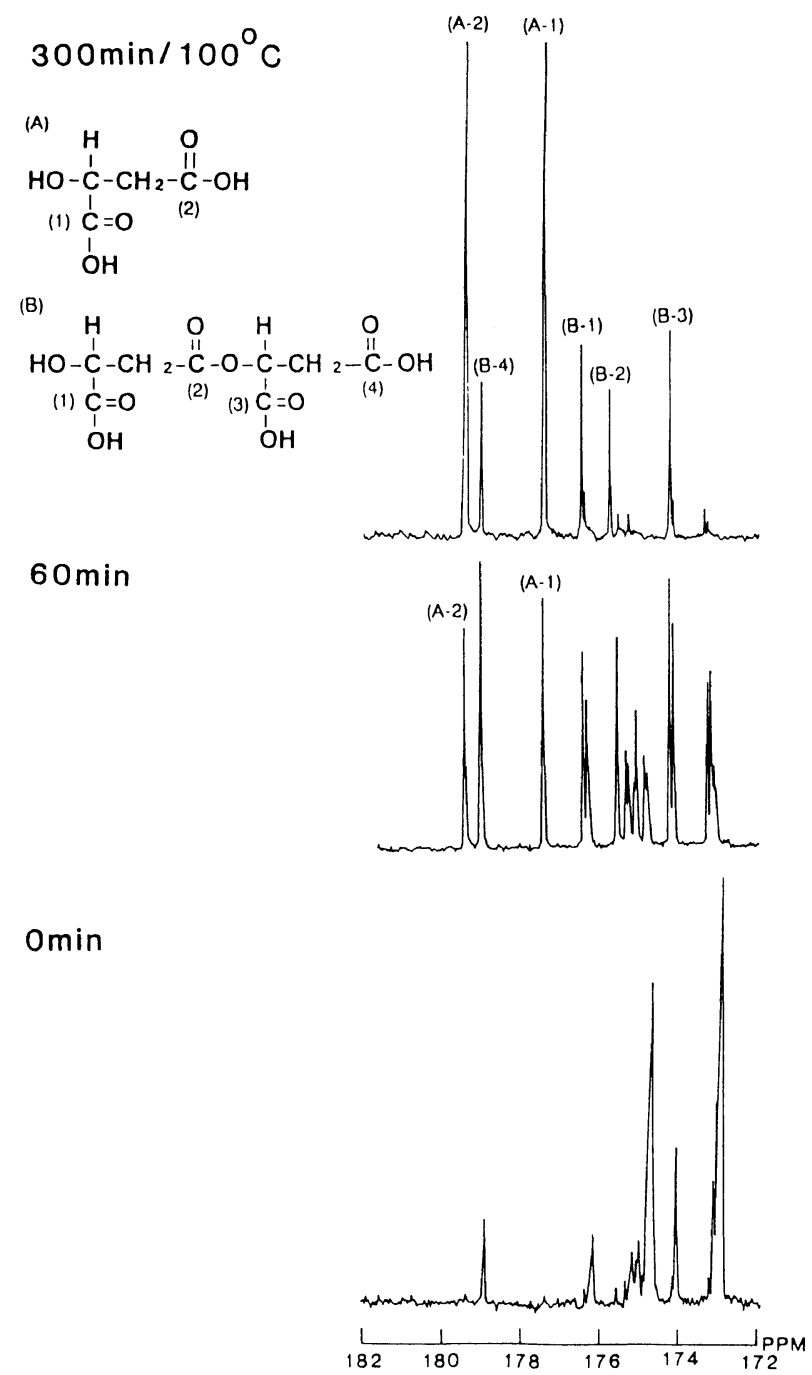

(3)

(2)

(1)

Figure 6. $67.94 \mathrm{MHz}{ }^{13} \mathrm{C}$ NMR spectra at lower magnetic field at different stages of auto-catalytic cleavage of poly(L-malic acid): $t=0 \mathrm{~min}$ compared with that of $t=60 \mathrm{~min}$ and $t=300 \mathrm{~min}$.

Confirmation of Optical Purity of the Cleavage

\section{Products}

The optical purity of the cleavage products as L-malic acid was assayed by an enzymatic titration using a pair of diagnostic kits for both of possible optical isomers as L-malic acid and D-malic acid. It was confirmed that both auto-catalytic cleavage and alkaline hydrolysis reaction of the $\operatorname{poly}(\beta$-L-malic acid) gave only L-malic acid as the decomposition products and that racemization did not take place during the course of decomposition.

\section{CONCLUSION}

Taking into account of the absence of change in methylene chemical shifts in the ${ }^{1} \mathrm{H}$ NMR spectra, with the significant conformational differences of the polymer chains of $\mathrm{Ca}$-ion bound and $\mathrm{Ca}$-ion freed poly(L-malic acid) respectively, the primary structure was deduced as poly( $\beta$-L-malic acid). Assignments 


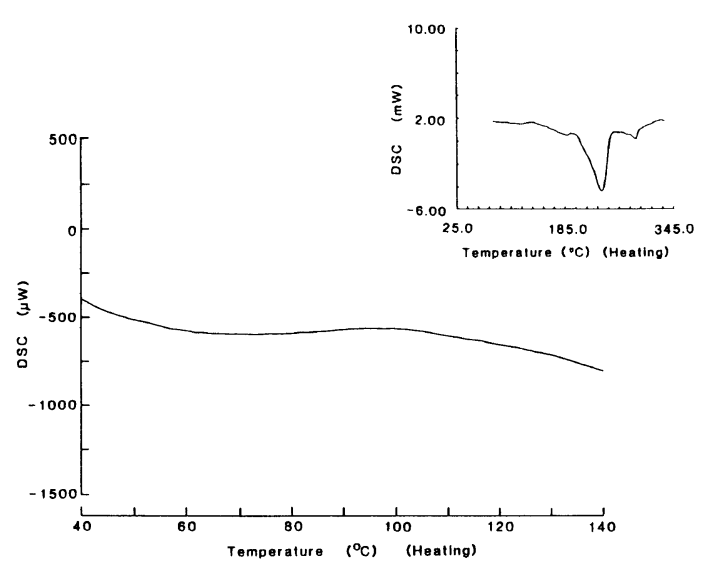

Figure 7. DSC of the solid state $\operatorname{poly}(\beta$-L-malic acid $)$.

of fine structures of both ${ }^{1} \mathrm{H}$ and ${ }^{13} \mathrm{C}$ NMR spectra could be attained comparing the spectrum recorded at different stage of cleavage via auto-catalytic hydrolysis of the polymer in aqueous solution. The polymer is highly hygroscopic and auto-catalytically cleaves in aqueous solution. The auto-catalytic cleavage reaction could be accelerated on heating the aqueous solution, for example, after $300 \mathrm{~min}$ heating at $100^{\circ} \mathrm{C}$ yielded L-malic acid as $98 \%$ of the polymer.
Detailed analysis of ${ }^{13} \mathrm{C}$ NMR spectra enables us to confirm the primary structure as being composed of not only linear chains but also polymer with branched chains. DSC on the solid state polymer shows neither glass transition temperature nor melting temperature below the decomposition temperature which starts at $185^{\circ} \mathrm{C}$ accompanied with endothermic reactions.

Enzymatic titration using a diagnostic kits applied for the cleaved products confirmed that racemization does not take place during the course of decomposition of the $\operatorname{poly}(\beta$-Lmalic acid) via hydrolytic cleavages either auto-catalytic or alkaline hydrolysis.

\section{REFERENCES}

1. N. Nagata, T. Nakahara, and T. Tabuchi, Biosci. Biotech. Biochem., in preparation.

2. N. Ohtani, Y. Kimura, and T. Kitao, Kobunshi Ronbunshu, 44, 701 (1987).

3. H. Fischer, S. Erdmann, and E. Holler, Biochemistry, 28, 5219 (1989).

4. T. Ouchi and A. Fujino, Makromol. Chem., 190, 1523 (1989). 